\title{
Factores psicosociales y sociodemográficos vinculados al uso de sustancias psicoactivas en mujeres durante el embarazo. Una revisión narrativa
}

\author{
Lourdes María López-Granados', Cristina de J. Cruz-Cortés' \\ ' Departamento de Investigación Psicosocial y Documental. Centros de Integración Juvenil, A.C., México.
}

\begin{abstract}
RESUMEN
Introducción: el consumo de sustancias durante el embarazo, sus efectos y consecuencias son un problema social y de salud pública grave y poco visible. Debido a los riesgos y consecuencias, se vuelve importante visibilizar los factores de riesgo del consumo de drogas en este grupo poblacional. Objetivo: identificar factores psicosociales y sociodemográficos del consumo de drogas lícitas e ilícitas en mujeres durante el embarazo. Método: se realizó una búsqueda de artículos de investigación, publicados entre 2010 y 2016, que trataran sobre estos factores en relación con el uso de drogas legales e ilegales durante el embarazo. Resultados: se encontraron 86 artículos, de los cuales se eliminaron los relacionados con efectos y consecuencias médicas y los realizados en animales. Después de la depuración, se concentraron 30 artículos, en los que se identificaron factores como edad, condiciones educativas, familiares, sociales, económicas, características del embarazo y antecedentes del mismo, así como la autopercepción de vulnerabilidad y la actitud favorable hacia el consumo de sustancias durante la gestación. Conclusiones y discusión: es necesario dedicar más esfuerzo al conocimiento de esta problemática y direccionar la búsqueda de raíces sociales complejas, sobre todo en el embarazo de adolescentes y otras poblaciones vulnerables.
\end{abstract}

Palabras clave: embarazo, factores de riesgo, uso de alcohol, uso de tabaco, uso de drogas.

\begin{abstract}
Introduction: substance use during pregnancy is a serious and little visible social and public health problem. Due to the risks and effects it is necessary to recognize the risk factor for drug use in this population group. Objective: to identify psychosocial and socio-demographic risk factors for drug use during pregnancy. Method: a research for articles published from 2010 to 2016 focused on psychosocial and socio-demographic factors correlated with the use of legal and illegal drugs during pregnancy was carried out. Results: 86 articles were found and some were removed from the results as they focused on basic research or medical consequences. 30 articles were considered and factors such as age, formal education, characteristics of pregnancy, positive attitudes towards substance use in pregnancy and family, social and economic conditions were identified. Conclusions and discussion: it is necessary to continue the research in this problem, specially focused in complex social factors and vulnerable populations.
\end{abstract}

Keywords: pregnancy, risk factors, alcohol abuse, smoking, drug abuse.

\footnotetext{
Autora de correspondencia:

Lourdes María López-Granados. Zempoala Núm. 77, col. Narvarte, del. Benito Juárez, C.P. 03600, Ciudad de México. Correos electrónicos: lourdes.lopez@cij.gob.mx; loulopezgranados@hotmail.com

Recibido: 11 de octubre de 2017.

Aceptado: 30 de octubre de 2017.

DOI: 10.28931/riiad.2017.2.06
} 


\section{INTRODUCCIÓN}

La prevalencia del uso de sustancias adictivas es mayor en los hombres que en las mujeres, pero esta brecha de género se está reduciendo de forma constante $(\mathrm{McHu}$ gh, Wigderson, \& Greenfield, 2014; Ortiz, Soriano, Meza, Martínez, \& Galván, 2006). La expansión del consumo de drogas ha alcanzado a las mujeres en edad fértil, lo que ha generado diversos retos médicos y sociales (Kasada, Marconi, Pagliarini, \& Rossi, 2013).

Los efectos y consecuencias del consumo de sustancias son distintos en hombres y mujeres por las características fisiológicas particulares de ambos (Cruz, 2014). En las mujeres, en relación con los hombres, la absorción, la unión a proteínas, la distribución y el metabolismo de sustancias puede ser diferente, debido a la eficiencia de algunas enzimas, la composición corporal (la relación tanto de agua como de grasa), el metabolismo cerebral, la influencia hormonal en las funciones fisiológicas y las variaciones en las respuestas a las drogas, de acuerdo con la fase del ciclo menstrual o la edad (National Institute on Drug Abuse [NIDA], 2015).

Las mujeres sufren con más frecuencia efectos secundarios derivados del consumo de algunas sustancias (Thürmann \& Hompesch, 1998). Si bien se requiere mayor investigación al respecto, información reciente (NIDA, 2015) sugiere que ellas pueden ser más susceptibles a las consecuencias médicas, psiquiátricas y sociales del uso de drogas. En comparación con los hombres, las mujeres tienen una latencia más corta desde el inicio del consumo de sustancias hasta la aparición de trastornos por el uso de éstas y lo presentan de forma más severa. Además, las que sufren problemas por consumo de sustancias presentan mayor prevalencia de trastornos comórbidos (Marín-Navarrete, et al., 2015).

Las mujeres reportan mayor deterioro en los ámbitos laboral, social, psiquiátrico y médico, en relación con los hombres. En cuestión de tratamiento, muestran tasas más altas de abandono (Marín-Navarrete et al., 2015) y es más probable que recaigan después de recibir atención especializada (NIDA, 2015).

Esta diferencia en la repercusión del consumo de drogas puede deberse, en parte, a las formas de vida, los roles, las funciones y las expectativas que les son encomendados social y culturalmente (Berenzon, Romero, Tiburcio, Medina-Mora, \& Rojas, 2007; NIDA, 2015). La estigmatización de las mujeres con problemas de adicción a las drogas (Castillo \& Gutiérrez, 2008) refuerza el aislamiento social, lo que a la vez favorece el ocultamiento del problema, la ausencia de petición de ayuda para superar el mismo, el uso de más sustancias con la finalidad de manejar estas dificultades (Ortiz et al., 2006) y la demora de la solicitud de tratamiento, hasta el punto en que las consecuencias sobre su salud física y mental o en su vida familiar, social o laboral, resultan insostenibles.

$\mathrm{Si}$ a las situaciones anteriores, se agrega el embarazo, el consumo de sustancias se presenta como un fenómeno de especial preocupación tanto para la salud del feto en desarrollo-debido a los efectos teratogénicos de las sustancias adictivas y a sus consecuencias negativas- como para las condiciones de vida posteriores al nacimiento.

El consumo de sustancias psicoactivas durante el embarazo no es un fenómeno aislado, está influido por el entorno cultural y social: con él se combinan otras circunstancias adversas como pobreza, discriminación, abandono educativo, falta de empleo, violencia interpersonal, desintegración familiar, antecedentes de abuso -físico y sexual- y, en general, un inadecuado cuidado de la salud (Romero \& Gómez, 1997; Wright, Schuetter, Fombonne, Stephenson, \& Haning 2012). Resalta, además, que los mecanismos institucionales de tratamiento integral -tanto para las mujeres como para sus hijos- no se encuentran disponibles, sobre todo en países en vías de desarrollo, lo que abona a la situación vulnerable de ambos.

Una condición que requiere especial atención es el consumo de sustancias en adolescentes embarazadas, una población particularmente vulnerable; los productos de embarazos adolescentes presentan mayor riesgo de prematuridad, de morbilidad y retraso en el crecimiento, todo ello en independencia de los factores sociodemográficos y la exposición prenatal a las sustancias (Cornelius, Goldschmidt, Day, \& Larkby, 2002).

El consumo de drogas durante el embarazo es una temática de interés para la comunidad científica en varios sentidos, desde el conocimiento de los efectos de las diferentes sustancias sobre el producto a corto, mediano y largo plazo, hasta la salud física (en muchos casos con problemas crónicos) y mental de las madres y de sus hijos, pasando por los requerimientos de atención médica y de salud mental de ambos. Además, las situaciones éticas y jurídicas que se ponen de manifiesto con esta problemática, evidencian que el consumo de sustancias psicoactivas durante el embarazo es un problema social y de salud pública grave y poco visible que supone un reto para las instituciones de salud, debido a las necesidades de contención y atención multidisciplinaria durante el embarazo, en el transcurso a la maternidad y en los años posteriores.

Debido a los riesgos, las consecuencias y demás condiciones, resulta necesario compilar y contribuir al conocimiento de los factores de riesgo del consumo de drogas específicos de este grupo poblacional. Dicha información puede apoyar el desarrollo de estrategias in- 
tegrales con intervenciones eficaces y específicas para subsanar las necesidades clínicas de estas mujeres y sus productos; se contribuirá, además, al desarrollo de políticas públicas que ayuden a reducir otros riesgos como la ruptura familiar, el abuso infantil, los ciclos intergeneracionales de desventaja y los problemas de salud física y mental de las mujeres y sus hijos.

\section{MÉTODO}

Se realizó una revisión narrativa, mediante la consulta de las siguientes bases de datos: Consorcio Nacional de Recursos de Información Científica y Tecnológica (CONRICYT), Directory of Open Access Journals, DynaMed, EBSCO, PubMed, PsycINFO, REDALyC, Science Direct y las Bibliotecas Virtuales de la Universidad Autónoma Metropolitana y la Universidad Nacional Autónoma de México. Las búsquedas se realizaron utilizando como palabras clave: "pregnancy", "risk factors", "drugs", "drug abuse", "alcohol", "tobacco", "marijuana", "cocaine"-así como sus equivalentes en español- y diversas combinaciones entre ellas; la investigación se limitó a artículos publicados entre 2010 y 2016.

Una primera pesquisa arrojó un total de 62 artículos clasificados por substancias, en un segundo momento se localizaron 24 más. Al final, se realizó la recuperación de artículos referenciados por los primeros artículos seleccionados, siempre y cuando cumplieran con los criterios de búsqueda.

Se incluyeron artículos que:

- Abordaran los factores psicosociales y sociodemográficos asociados con el consumo de sustancias por mujeres durante el embarazo (sin restricción en las edades de las participantes).

- Tuvieran un corte cualitativo, cuantitativo o fueran revisiones sistemáticas.

- Estuvieran escritos en inglés o en español.

- Fueron publicados entre 2010 y 2016.

Se excluyeron aquellos que:

- Presentaran el embarazo como el resultado de conductas de riesgo.

- Abordaran únicamente el aspecto médico, farmacológico o bioquímico del consumo de sustancias durante el embarazo y sus consecuencias.

- Se centraran en tratamientos médicos y psicológicos, tanto para la madre como para el producto.

- No expusieran factores psicosociales o sociodemográficos vinculados con el consumo de sustancias durante el embarazo.
- Hubieran sido publicados fuera del lapso elegido.

- No proporcionaran el acceso total al contenido del artículo.

Se encontraron 86 artículos de investigación. No se realizó ninguna evaluación de la calidad científica de los artículos seleccionados.

Después de efectuar una evaluación inicial por títulos, fechas de publicación, temáticas y resúmenes, se excluyeron todos aquellos que no cumplían con los criterios de inclusión, así como los duplicados; al final, se consideraron 30 artículos (ver Figura 1).

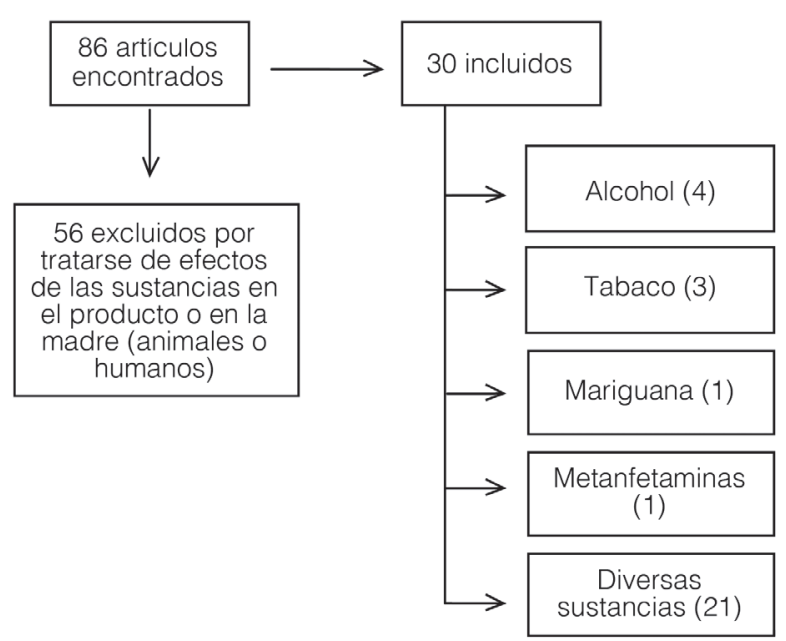

Figura 1. Diagrama de flujo de la búsqueda

Resalta el hecho de haber encontrado pocos artículos que abordaran el fenómeno de forma directa; de ellos un pequeño porcentaje se encuentra en español y una minoría involucra población mexicana.

Para facilitar el manejo de la información, los artículos se agruparon de acuerdo con la sustancia en la que se enfocaban: alcohol, tabaco, mariguana o varias de ellas.

De cada artículo se recopiló la siguiente información: autores, título, año de publicación, país en el que se desarrolló el estudio, tipo de estudio, objetivos, población/ muestra, procedimientos, resultados y conclusiones. Para el manejo y el almacenaje de la información se empleó el programa Microsoft Excel.

A continuación se presentan los principales factores sociodemográficos y psicosociales vinculados con el uso de drogas durante el embarazo; se conservó la división por sustancias: alcohol, tabaco, mariguana, metanfetaminas y varias de ellas. 


\section{RESULTADOS}

\section{Estudios relacionados con el consumo de alcohol y tabaco durante el embarazo}

Se agruparon los resultados de los estudios que tratan el consumo de alcohol y tabaco durante el embarazo, pues un gran porcentaje de artículos los abordan en conjunto, esto puede deberse a las condiciones de legalidad y de consumo social/aceptado, lo que puede incidir en mayores muestras.

Los factores identificados que inciden en el consumo de alcohol y tabaco en el embarazo podrían categorizarse en familiares, de salud y sociodemográficos. Entre los primeros se encontraron antecedentes de consumo de alcohol en las familias de origen, la falta de una pareja estable o tener una con problemas legales, así como reportar una mayor cantidad de parejas sexuales (Ceccanti et al., 2014; Desmond, et al., 2012; Veloso \& Monteiro, 2013).

En cuanto a los aspectos sanitarios, se encontró relación con el menor número de gestaciones previas y el consumo de alcohol y tabaco en las mismas; con la falta de conocimiento sobre los efectos adversos del consumo; los intentos de interrupción del embarazo en curso; una débil adherencia al control prenatal; una pobre autopercepción de salud; y actitudes favorables al consumo en la gestación (Ceccanti et al., 2014; Cui, Shooshtari, Forget, Clara, \& Cheung, 2014; Desmond, et al., 2012; Passey, Sanson-Fisher, D'Este, \& Stirling, 2014; Singh, Mini, \& Thankappan, 2015; Veloso \& Monteiro, 2013).

En cuanto a la última categoría, factores sociodemográficos, el consumo se asoció con ser residentes de zonas urbanas, tener bajos ingresos económicos y una menor escolaridad, reportar una menor o nula adherencia religiosa, ser víctimas de violencia y tener un apoyo social significativo que las acerca al consumo de sustancias. Conviene resaltar que respecto a la edad se encontraron diferencias, mientras un estudio reportó como factor de riesgo una mayor edad en la madre, otros lo reportan como un factor protector (Ver Tabla 1; Cui et al., 2014; Desmond, et al., 2012; Passey et al., 2014; Singh et al., 2015; Veloso \& Monteiro, 2013).

\section{Consumo de mariguana y metanfetaminas}

Como se muestra en la Tabla 2, sólo se encontró un estudio enfocado en el consumo exclusivo de mariguana; señala que la edad materna es un factor determinante para el uso de esta droga, pues a menor edad se reporta un mayor consumo (De Genna, Cornelius, Goldschmidt, \& Day, 2015).
Dentro de los parámetros de búsqueda se localizó un artículo relacionado con el consumo de metanfetaminas durante el embarazo, en cuyos resultados se reportan como factores asociados al consumo un inadecuado funcionamiento familiar y antecedentes familiares tanto de alcoholismo como de suicidios (Barlow et al. 2010).

\section{Consumo de diversas sustancias}

Los artículos integrados en este apartado consideraron factores sociodemográficos como el estado civil, la edad y el ingreso económico. Encontraron relación entre el consumo de diversas sustancias en el embarazo y la soltería, una menor edad y la presencia de condiciones socioeconómicas adversas (bajo nivel de ingresos en la familia, inseguridad económica y la falta de un hogar). Respecto a la educación se encontraron contradicciones, pues tanto niveles bajos como altos de escolaridad, se identificaron como factores de riesgo. La religión ya sea la afiliación o la práctica habitual- es otro factor frecuentemente mencionado -en ocasiones como factor protector, en otras como elemento que propicia un mayor consumo de sustancias entre seguidores de algunas creencias-. Se encontraron, además, diferencias en raza, origen étnico y situación migratoria (ver Tabla 3; Ali, Davis, Simmons, \& Ramroop, 2014; Assanangkornchai, Saingam, Apakupakul, \& Edwards, 2016; Bessa et al., 2010; Bottorff et al., 2014; Davie-Gray, Moor, Spencer, \& Woodward, 2013; De Santis et al., 2011; Lamy \& Thibaut, 2010; Muckle et al., 2011; Onah, Field, van Heyningen, \& Honikman, 2016; Peña \& Matute, 2010; van Gelder et al., 2010; Wickersham et al., 2016).

Entre los factores familiares relacionados con el consumo de sustancias en el embarazo se encuentran el tener una pareja de mayor edad, el uso de drogas por parte de la pareja y otros familiares, la presencia de mayor consumo -tanto en frecuencia, como en cantidad- de alcohol previo al embarazo y los antecedentes de actividades delictivas. También se encontró relación con haber sufrido abandono o abuso físico y sexual en la infancia, y haber sido víctima de violencia interpersonal y doméstica (Ali et al., 2014; Barnes, Khaled, \& Crome, 2010; Bjønness, 2015; De Santis et al., 2011; Muckle et al., 2011; Onah et al., 2016; Rodrigues et al., 2014, Wickersham et al., 2016).

Las condiciones de salud, tanto general como sexual y reproductiva, con las que se encontró relación fueron: problemas crónicos de salud, trastornos psiquiátricos comórbidos, antecedentes de problemas obstétricos, embarazos no planificados, intención de aborto, menor número de embarazos y, aunque no es problema de salud, se reportó la falta de apoyo y ayuda por parte del sistema de salud, así como la falta de respuesta 

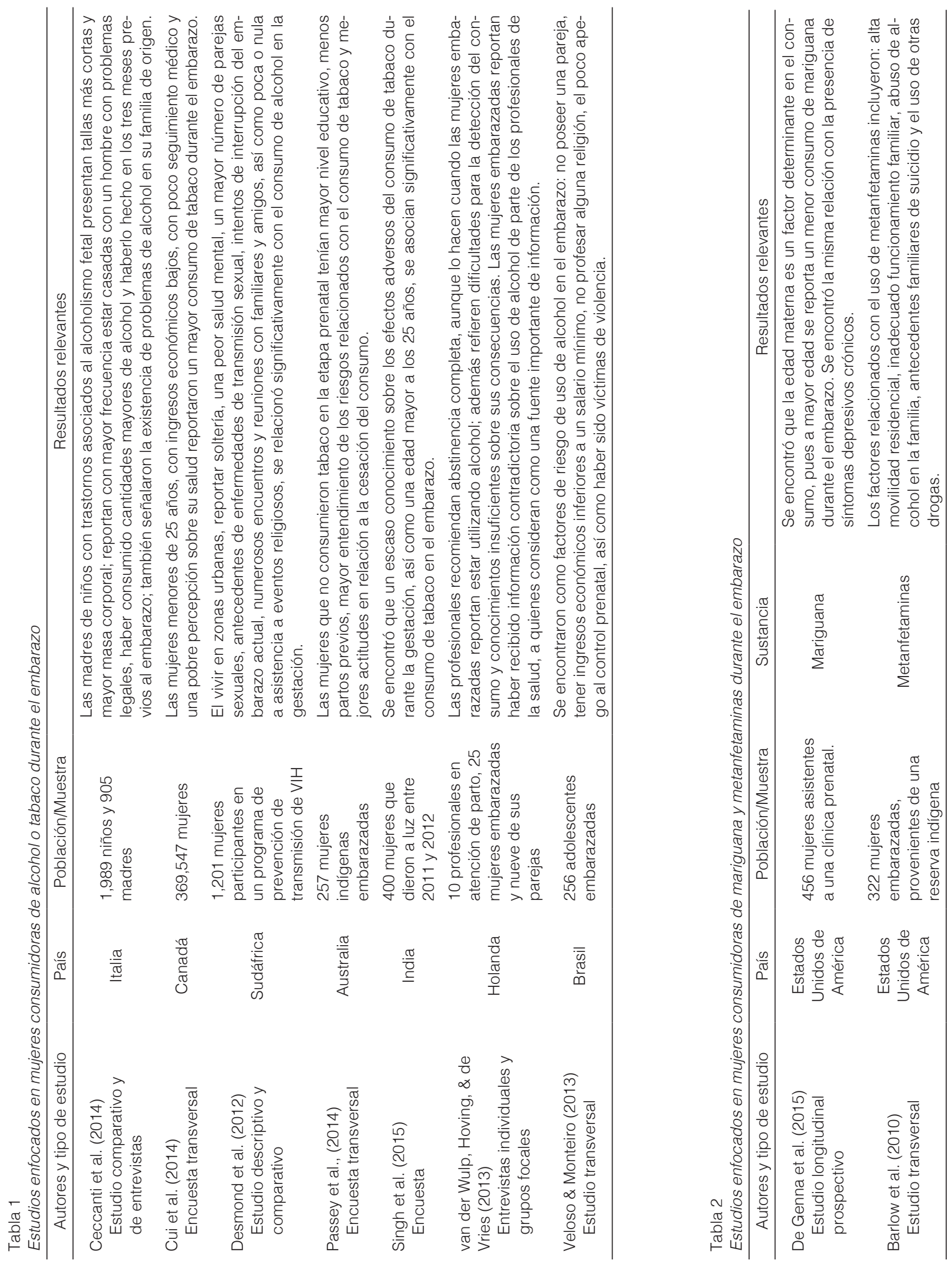


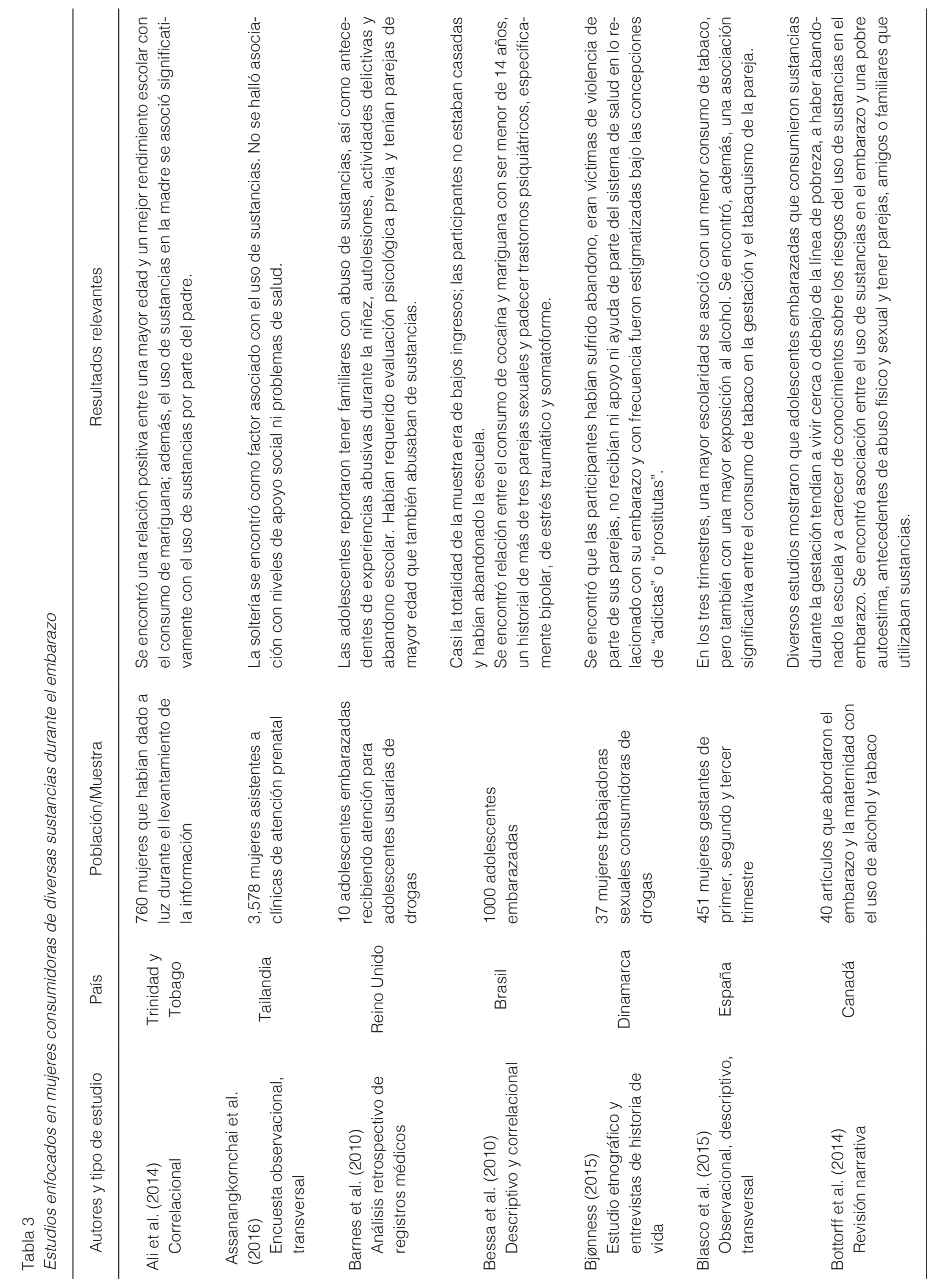




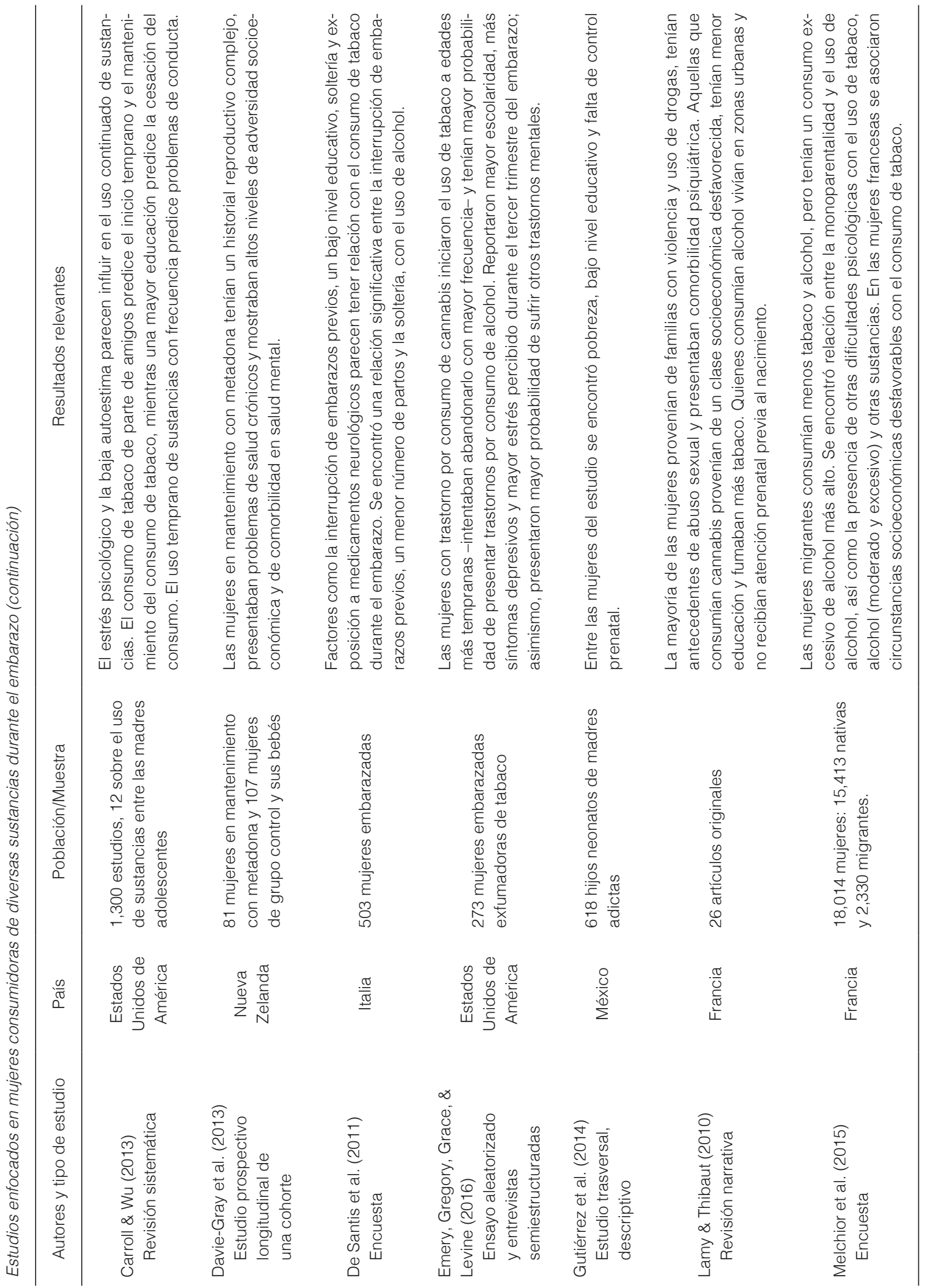




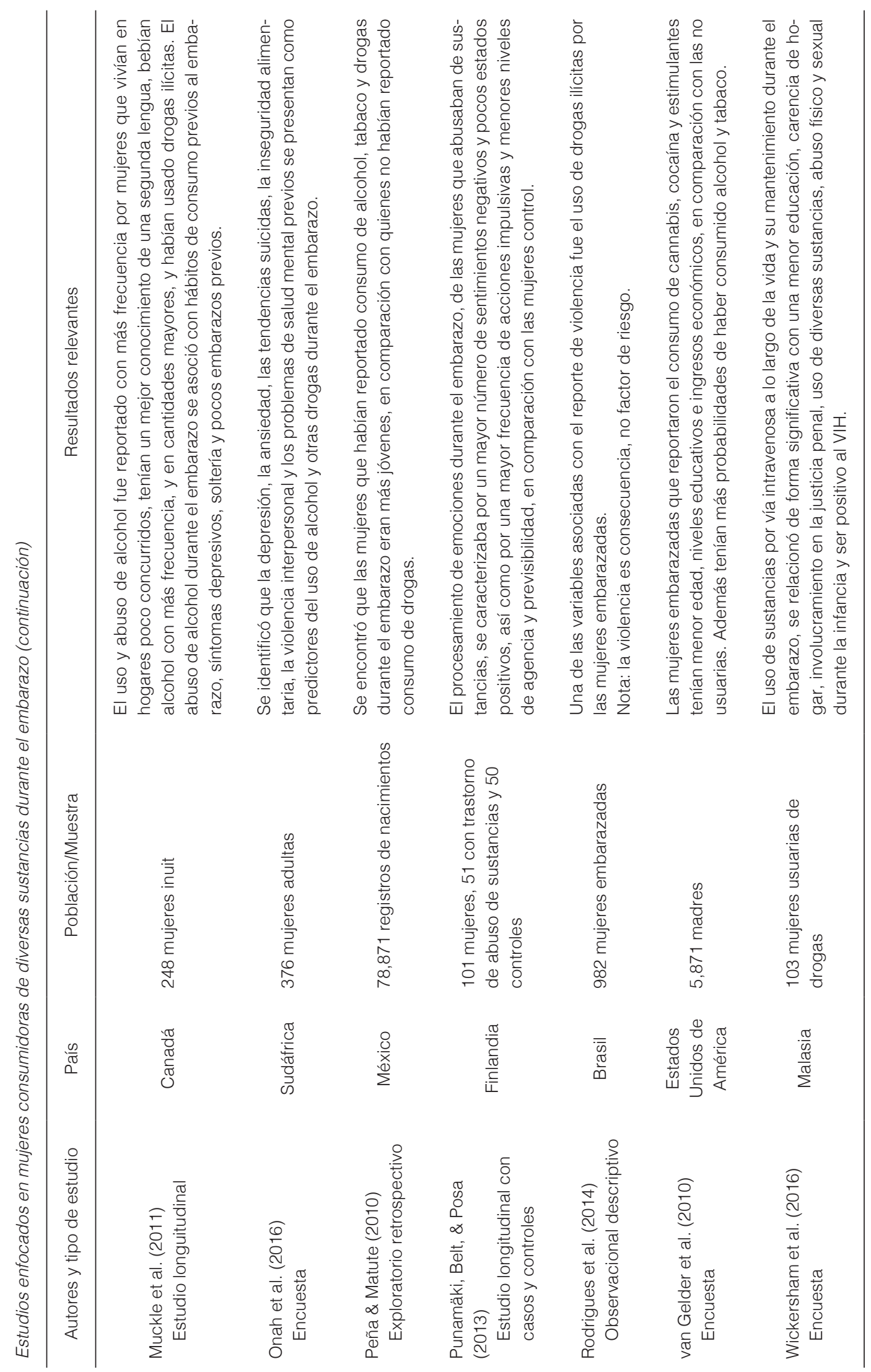


institucional a esta problemática (Assanangkornchai et al., 2016; Bessa et al., 2010; Bjønness, 2015; Bottorff et al., 2014; Davie-Gray et al., 2013; De Santis et al., 2011).

\section{DISCUSIÓN Y CONCLUSIONES}

El objetivo de esta revisión fue identificar los factores de riesgo relativos al consumo de sustancias psicoactivas en el embarazo, en artículos publicados entre 2010 y 2016.

Entre los antecedentes familiares se encontraron el consumo de alcohol en las familias de origen, la falta de una pareja estable o tener una de mayor edad, reportar una mayor cantidad de parejas sexuales o estar con alguien con problemas legales o de consumo de sustancias psicoactivas (Ceccanti et al., 2014; Desmond, et al., 2012; Veloso \& Monteiro, 2013); otros factores de riesgo fueron la disfuncionalidad familiar y los antecedentes familiares de diferentes problemáticas de salud mental, entre los que resaltó la pérdida de familiares por suicidio (Barlow et al., 2010).

En relación con los factores sociodemográficos se mencionó la edad materna, sobre la que se encontraron disyuntivas, pues mientras un estudio reportó como factor de riesgo una mayor edad en la madre, otros la señalaron como factor protector (Cui et al., 2014; Desmond, et al., 2012; Passey et al., 2014; Singh et al., 2015; Veloso \& Monteiro, 2013). El consumo de drogas durante el embarazo se relacionó, además, con la presencia de condiciones socioeconómicas adversas, como un bajo nivel de ingresos en la familia, inseguridad económica y la falta de un hogar). Respecto al nivel educativo, se hallaron contradicciones, pues tanto niveles bajos como altos de escolaridad se identificaron como factores de riesgo. La religión, como afiliación y como práctica habitual, es otro factor frecuentemente mencionado (en ocasiones como factor protector, en otras identificado con un mayor consumo de sustancias en algunos grupos). Se encontraron, además, diferencias en raza, origen étnico y situación migratoria (Ali et al., 2014; Assanangkornchai et al., 2016; Bessa et al., 2010; Bottorff et al., 2014; Davie-Gray et al., 2013; De Santis et al., 2011; Lamy \& Thibaut, 2010; Muckle et al., 2011; Onah et al., 2016; Peña \& Matute, 2010; van Gelder et al., 2010; Wickersham et al., 2016).

Respecto a los factores de salud se identificaron un menor número de gestaciones previas, antecedentes de problemas obstétricos, embarazos no planificados, intención de aborto, y consumo de alcohol y tabaco, intentos de interrupción del embarazo en curso, una débil adherencia al control prenatal y una pobre autopercepción de salud (Ceccanti et al., 2014; Cui et al., 2014; Desmond, et al., 2012; Passey et al., 2014; Singh et al., 2015; Veloso \& Monteiro, 2013).
Entre los factores psicosociales se pueden mencionar la autopercepción de inestabilidad emocional, una actitud favorable al consumo de sustancias psicoactivas durante la gestación o en forma previa al embarazo (a lo que se añade la falta de conocimiento sobre los efectos adversos del consumo). También se encontró relación con haber sufrido abandono y abuso físico y sexual en la infancia, así como con haber sido víctima de violencia interpersonal y doméstica (Ali et al., 2014; Barnes et al., 2010; Bjønness, 2015; De Santis et al., 2011; Muckle et al., 2011; Onah et al., 2016; Rodrigues et al., 2014; Wickersham et al., 2016).

Destacó, también, la carencia de apoyo y ayuda por parte del sistema de salud y la falta de respuesta institucional ante este problemática (Assanangkornchai et al., 2016; Bessa et al., 2010; Bjønness, 2015; Bottorff et al., 2014; Davie-Gray et al., 2013; De Santis et al., 2011).

Es reducido el material especializado encontrado sobre la materia, al parecer porque el tema fue abordado previamente, sobre todo en la década de los noventa; esto se infiere pues en la búsqueda se encontraron artículos publicados con anterioridad a la fecha estipulada en los criterios de inclusión.

Esto resulta de interés porque implica la oportunidad y pertinencia de la actualización, sobre todo si se considera el incremento en el número de embarazos en población adolescente (Grupo Interinstitucional para la Prevención del Embarazo en Adolescentes, 2017) y el aumento en el consumo de sustancias psicoactivas por parte de las mujeres, reportado en las encuestas en hogares y en población educativa de nivel medio superior y superior (Instituto Nacional de Psiquiatría Ramón de la Fuente Muñiz [INPRFM], Comisión Nacional contra las Adicciones [CONADIC], \& Secretaría de Salud [SS], 2015; Instituto Nacional de Salud Pública, INPRFM, CONADIC, \& SS, 2016).

Estos datos dejan ver la futura intersección entre estas dos condiciones y las problemáticas que pueden acarrear para su atención desde la política pública. Es de destacarse la relativa escasez de estudios e investigaciones realizados en América Latina y, particularmente, en México, sobre todo si se considera que el consumo de drogas durante el embarazo es un problema poblacional que está cobrando cada vez más importancia, pues afecta la salud, la educación, la economía y las relaciones sociales y culturales (Grupo Interinstitucional para la Prevención del Embarazo en Adolescentes, 2017).

La investigación en este campo en el periodo estudiado se enfocó, en forma primaria, en evaluaciones cuantitativas, de prevalencia y patrones de consumo de sustancias durante el embarazo. La indagación en estos 
estudios ha sido básicamente descriptiva, transversal y a través de encuestas, con tendencia a identificar predictores del uso de sustancias durante el embarazo en poblaciones específicas (indígenas, neonatos en terapia intensiva, adolescentes). Hay estudios realizados a partir de expedientes o registros hospitalarios. La mayoría de ellos se efectuaron a través de autoinformes, escalas con indicadores de consumo, exámenes de orina, meconio o cabello de la madre.

El consumo de sustancias psicoactivas parece ser frecuente, en diversas proporciones, entre las mujeres y en el embarazo (Peña \& Matute, 2010). No obstante, es necesario contar con mayor evidencia e indagar el consumo de sustancias ante eventos específicos como el aborto o el nacimiento de niños en condiciones de salud física comprometida, así como en poblaciones en migración o en adolescentes, por citar algunos casos de vulnerabilidad.

Hay una clara necesidad de realizar aproximaciones preventivas o terapéuticas integrales y efectivas que eviten con anticipación o atiendan el consumo de sustancias por parte de las madres durante el embarazo. Es preciso preparar a médicos obstetras, pediatras, neonatólogos, parteras y enfermeras, para poder realizar una oportuna detección e intervención con las mujeres en las consultas de control prenatal (Blasco et al., 2015; Ceccanti et al, 2014; De Genna et al., 2015; Peña \& Matute, 2010); sin embargo, también existe la necesidad urgente de contrarrestar las condiciones sociales, familiares e institucionales de atención de esta población vulnerable (Ceccanti et al., 2014); es preciso educar sobre las consecuencias del consumo de alcohol, tabaco y otras drogas durante esta etapa de vida, así como ofrecer intervenciones en las que se brinde información sobre los daños y consecuencias específicas del consumo de sustancias psicoactivas en las mujeres.

Todas estas brechas necesitan ser visualizadas tanto en la investigación, como en la prevención y la atención (Bessa, et al., 2010); se debe considerar que las mujeres que informan sobre el uso de sustancias durante el embarazo difieren en el fondo demográfico, familiar, social, de comorbilidad física y psiquiátrica y de atención institucional, por lo que requieren de tratamiento y rehabilitación especializados, que den seguimiento a todas las condiciones, preferencias, necesidades y requerimientos pertinentes, por lo que en particular sería necesario indagar cómo son atendidas y si existen protocolos de intervención ya establecidos por organismos internacionales (Bottorf et al., 2014; van Gelder et al., 2010).

\section{FUENTES DE FINANCIAMIENTO}

Este estudio no contó con fuentes de financiamiento.

\section{CONFLICTO DE INTERÉS}

Esta investigación no presenta conflicto de interés respecto a los datos presentados.

\section{REFERENCIAS}

Ali, Z., Davis, G., Simmons, V., \& Ramroop, R. (2014). Substance use in pregnancy at the Mt Hope Women's Hospital in Trinidad. West Indian Medical Journal Open, 1(1), 18-21. doi:10.7727/ wimjopen.2012.098

Assanangkornchai, S., Saingam, D., Apakupakul, N., \& Edwards, G. (2016). Alcohol consumption, smoking, and drug use in pregnancy: prevalence and risk factors in Southern Thailand. AsiaPacific Psychiatry, 9(1), e12247. doi: 10.1111/appy.12247

Barlow, A., Mullany, B. C., Neault, N., Davis, Y., Billy, T., Hastings, R., ... \& Walkup, J. T. (2010). Examining correlates of methamphetamine and other drug use in pregnant American Indian adolescents. American Indian and Alaska native Mental Health Research, 17(1), 1-24. doi: 10.5820/aian.1701.2010.1

Barnes, W., Khaled, M., \& Crome, I., (2010). Triply troubled: criminal behavior and mental health in a cohort of teenage pregnant substance misusers in treatment. Criminal Behaviour and Mental Health, 20(5), 335-348. doi:10.1002/cbm.776

Berenzon, S., Romero M., Tiburcio M., Medina-Mora, M., \& Rojas, E. (2007). Riesgos asociados al consumo de alcohol durante el embarazo en mujeres alcohólicas de la Ciudad de México. Salud Mental, 30(1), 31-38

Bessa, M. A., Mitsuhiro, S. S., Chalem, E., Barros, M. C. de M., Guinsburg, R., \& Laranjeira, R. (2010). Correlates of substance use during adolescent pregnancy in São Paulo, Brazil. Revista Brasileira de Psiquiatria, 32(1), 66-69. doi:10.1590/s151644462010000100013

Bjønness, J. (2015). Narratives about necessity. Constructions of motherhood among drug using sex-sellers in Denmark. Substance Use \& Misuse, 50(6), 783-793. doi: 10.3109/10826084.2015.978648

Blasco, M., González, E., Gálvez, M., Lozano, I., Merino, F., Cuenca, F., ... Bellido, I. (2015). Exposure to tobacco, alcohol and drugs of abuse during pregnancy. A study of prevalence among pregnant women in Malaga (Spain). Adicciones, 27(2), 88-108.

Bottorff, L., Poole, N., Kelly, T., Greaves, L., Marcellus, L., \& Jung, M. (2014). Tobacco and alcohol use in the context of adolescent pregnancy and postpartum: a scoping review of the literature. Health and Social Care in the Community, 22(6), 561-574. doi: 10.1111/hsc. 12091

Carroll, S., \& Wu, L. (2013). Substance use among adolescent mothers: A review. Children and Youth Services Review, 35(5), 806 - 815. doi: 10.1016/j.childyouth.2013.02.004

Castillo, I., \& Gutiérrez, A. (2008) Consumo de drogas en mujeres asistentes a centros de tratamiento especializado en la Ciudad de México. Salud Mental, 31(5), 351-359.

Ceccanti, M., Fiorentino, D., Coriale, G., Kalberg, W., Buckley, D., \& May, P. (2014). Maternal risk factors for fetal alcohol spectrum 
disorders in aprovince in Italy. Drug and Alcohol Dependence, 145, 201-208. doi: 10.1016/j.drugalcdep.2014.10.017

Cornelius, M. D., Goldschmidt, L., Day, N. L., \& Larkby, C. (2002). Alcohol, tobacco and marijuana use among pregnant teenagers: 6-year follow-up of offspring growth effect. Neurotoxicology and Teratology, 24(6), 703-710. doi: 10.1016/S0892-0362(02)00271-4

Cruz, L. (2014). Diferencias entre sexos en los efectos de las drogas. $16^{\circ}$ Congreso Internacional en Adicciones. México: Centros de Integración Juvenil.

Cui, Y., Shooshtari, S., Forget, E., Clara, I., \& Cheung, K. (2014). Smoking during pregnancy: findings from the 2009-2010. Canadian Community Health Survey. Plos One, 9(1), e84640. doi: 10.1371/journal.pone.0084640

Davie-Gray, A., Moor, S., Spencer, C., \& Woodward, L. (2013). Psychosocial characteristics and poly-drug use of pregnant women enrolled in methadone maintenance treatment. Neurotoxicology and Teratology, 38, 46-52. doi: 10.1016/j.ntt.2013.04.006

De Genna, M., Cornelius, D., Goldschmidt, L., \& Day, L. (2015). Maternal age and trajectories of cannabis use. Drug and Alcohol Dependence, 156, 199-206. doi: 10.1016/j.drugalcdep.2015. 09.014

De Santis, M., de Luca, C., Mappa, I., Quattrocchi, T., Angelo, L., \& Cesari, E. (2011). Smoking, alcohol consumption and illicit drug use in an Italian population of pregnant women. European Journal of Obstetrics \& Gynecology and Reproductive Biology, 159(1), 106-110. doi:10.1016/j.ejogrb.2011.07.042

Desmond, K., Milburn, N., Richter L., Tomlinson, M., van Heerden, A., \& Rochtheram, M. (2012). Alcohol consumption among HIV-positive pregnant women in KwaZulu-Natal, South Africa: Prevalence and correlates. Drug and Alcohol Dependence, 120(1-3), 113-118. doi:10.1016/j.drugalcdep.2011.07.004

Emery, R. L., Gregory, M. P., Grace, J. L., \& Levine, M. D. (2016). Prevalence and correlates of a lifetime cannabis use disorder among pregnant former tobacco smokers. Addictive Behaviors, 54, 52-58. doi: 10.1016/j.addbeh.2015.12.008

Grupo Interinstitucional para la Prevención del Embarazo en Adolescentes. (2017). Estrategia Nacional para la Prevención del Embarazo en Adolescentes (ENAPEA). México: Gobierno Federal.

Gutiérrez, J., Padilla, H., Gutiérrez H., Barrera, J., Aguirre, O., Martínez, V., ... Ávalos, L. (2014). Evolución clínica de hijos de madres con adicciones internados en una unidad de terapia intensiva neonatal del Occidente de México. Ginecología y Obstetricia de México, 82, 441-447.

Instituto Nacional de Psiquiatría Ramón de la Fuente Muñiz, Comisión Nacional contra las Adicciones, \& Secretaría de Salud. (2015). Encuesta Nacional de Consumo de Drogas en Estudiantes 2014: Reporte de Drogas. México: Secretaría de Salud.

Instituto Nacional de Salud Pública, Instituto Nacional de Psiquiatría Ramón de la Fuente Muñiz, Comisión Nacional contra las Adicciones, \& Secretaría de Salud. (2016). Encuesta Nacional de Consumo de Drogas, Alcohol y Tabaco 2016-2017: consumo de drogas: prevalencias globales, tendencias y variaciones estatales. México: Secretaría de Salud.
Kasada, D., Marconi, S., Pagliarini, M., \& Rossi, R. (2013). Prevalence of drug abuse among pregnant women. Acta Paulista de Enfermagem, 26(5), 467-471. doi: 10.1590/S010321002013000500010

Lamy, S., \& Thibaut, F. (2010). Psychoactive substance use during pregnancy: a review. L'Encéphale, 36(1), 33-38. doi: 10.1016/j. encep.2008.12.009

Marín-Navarrete, R., de la Fuente-Martín, A., Cano-Arrieta, G., ViIlalobos-Gallegos, L., Bucay-Harari, L., Larios-Chávez, L., ... Ambriz-Figueroa, A. K. (2015). Mujeres con patología dual: características clínicas y de tratamiento. Revista Internacional de Investigación en Adicciones, 1(1), 41-49.

McHugh, K., Wigderson, S., \& Greenfield, S. (2014). Epidemiology of substance use in reproductive-age women. Obstetrics and Gynecology Clinics of North America, 41(2), 177-189. doi: 10.1016/j.ogc.2014.02.001

Melchior, M., Chollet, A., Glangeaud-Freudenthal, N., Saurel-Cubizolles, J., Dufourg, M., \& Sutter-Dallay, A. (2015). Tobacco and alcohol use in pregnancy in France: the role of migrant status. Addictive Behaviors, 51, 65-71. doi: 10.1016/j.addbeh.2015.07.015

Muckle, G., Laflamme, D., Gagnon, J., Boucher, O., Jacobson, J., \& Jacobson, S. (2011). Alcohol, smoking and drug use among inuit women of childbearing age during pregnancy and the risk to children. Alcoholism Clinical and Experimental Research, 35(6), 1081-1091. doi:10.1111/j.1530-0277.2011.01441.x.

National Institute on Drug Abuse. (2015) El consumo de sustancias en las mujeres. Recuperado de https://www.drugabuse.gov/es/ publicaciones/drugfacts/el-consumo-de-sustancias-en-lasmujeres

Onah, M., Field, S., van Heyningen, T., \& Honikman, S. (2016). Predictors of alcohol and other drug use among pregnant women in a peri-urban South African setting. International Journal of Mental Health Systems, 10(1) [10 pages]. doi: 10.1186/s13033016-0070-x

Ortiz, A., Soriano, A., Meza, D., Martínez, R., \& Galván, J. (2006). Uso de sustancias entre hombres y mujeres, semejanzas y diferencias. Resultados del sistema de reporte de información en drogas. Salud Mental, 29(5), 32-37

Passey, M. E., Sanson-Fisher, R. W., D’Este, C. A., \& Stirling, J. M. (2014). Tobacco, alcohol and cannabis use during pregnancy: Clustering of risks. Drug and Alcohol Dependence, 134, 44-50. doi:10.1016/j.drugalcdep.2013.09.008

Peña, J., \& Matute, E. (2010). Consumo de alcohol en mujeres embarazadas atendidas en el Hospital Civil de Guadalajara Dr. Juan I. Menchaca, entre 1991 y 1998. Espiral, Estudios sobre Estado y Sociedad, 16(47), 211-249.

Punamäki, R., Belt, R., \& Posa, T. (2013). Emotions during the transition to parenthood among substance abusing mothers: intensity, content and intervention effects. Journal of Reproductive and Infant Psychology, 31(3), 222-244. doi: 10.1080/02646838.2013.803046

Rodrigues, M., Moura, A., Seabra, M. T., Fernandes R., Leitão, L., Blima, L., ... Barbieri, M. A. (2014) Psychological violence against pregnant women in a prenatal care cohort: rates and associa- 
ted factors in São Luís, Brazil. BMC Pregnancie and Childbirth, 14(66), [09 pages]. doi: 10.1186/1471-2393-14-66

Romero, M., \& Gómez, C. (1997). La mujer embarazada con problemas de adicciones: consideraciones de género, investigación y tratamiento. Perinatología y Reproducción Humana, 11(4), 207-216. Recuperado de http://bases.bireme.br/cgi-bin/ wxislind.exe/iah/online/?IsisScript=iah/iah.xis\&src=google\&base =LILACS\&lang=p\&nextAction=Ink\&exprSearch=214315\&index Search $=$ ID

Singh, S., Mini, G., \& Thankappan, K. (2015). Tobacco use during pregnancy in rural Jharkhand, India. International Journal of Gynecology and Obstetrics, 131(2), 170-173. doi: 10.1016/j.ijgo. 2015.05.021

Thürmann, A., \& Hompesch, C. (1998). Influence of gender on the pharmacokinetics and pharmacodynamics of drugs. International Journal of Clinical Pharmacology and Therapeutics, 36(11), 586-590. Recuperado de http://europepmc.org/abstract/ med/9849747

van der Wulp, Y., Hoving, C., \& de Vries, H. (2013). A qualitative investigation of alcohol use advice during pregnancy: experiences of Dutch midwives, pregnant women and their partners. Midwifery, 29(11), e89-e98. doi: 10.1016/j.midw.2012.11.014 van Gelder, H., Reefhuis, J., Caton, R., Werler, M., Druschel, M., \& Roeleveld, N. (2010). Characteristics of pregnant illicit drug users and associations between cannabis use and perinatal outcome in a population-based study. Drug and Alcohol Dependence, 109(1-3), 243-247. doi:10.1016/j.drugalcdep.2010.01.007

Veloso, L., \& Monteiro C. (2013). Prevalencia y factores asociados al uso de alcohol en adolescentes embarazadas. Revista Latino-Americana de Enfermagem, 21(1) [09 pantallas]. Recuperado de http://docplayer.es/4587176-Prevalencia-y-factoresasociados-al-uso-de-alcohol-en-adolescentes-embarazadas $-1 . h t m l$

Wickersham, J., Loeliger, K., Marcus, R., Pillai, V., Kamarulzaman, A., \& Altice, F. (2016). Patterns of substance use and correlates of lifetime and active injection drug use among women in Malaysia. American Journal of Drug Alcohol Abuse, 42(1), 98-110. doi: 10.3109/00952990.2015.1101467

Wright, T. E., Schuetter, R., Fombonne, E., Stephenson, J., \& Haning, W. F. (2012). Implementation and evaluation of a harm-reduction model for clinical care of substance using pregnant women. Harm Reduction Journal, 9(1), 5. doi: 10.1186/1477-7517-9-5 